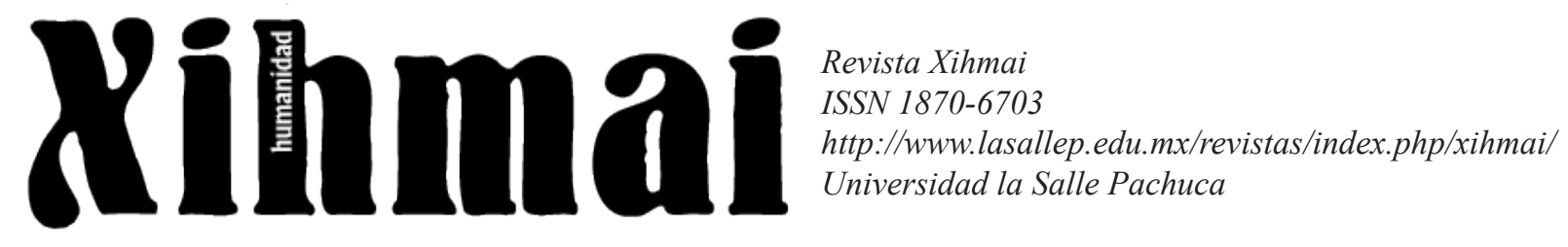

\author{
CIUDADANÍA Y EDUCACIÓN \\ CITIZENSHIP AND EDUCATION
}

Humberto Mejía Zarazúa

Universidad la Salle Pachuca

Nota sobre el autor

Ingeniero Agrícola y Maestro en Ciencias de la Educación. Colabora en la Dirección de Posgrado e Investigación y docente en las Maestrías de Educación (áreas Educación Superior y Administración Educativa y Gestión) en la Universidad La Salle Pachuca.

Esta investigación fue financiada con recursos del autor.

Recibido:1/1/2006

Aceptado:1/1/2006

Derechos de autor (C) 2006 Universidad La Salle Pachuca - Todos los derechos reservados.

Condiciones de Uso: La reproducción total o parcial de la obra en soporte electrónico queda prohibida sin la autorización de la Universidad la Salle Pachuca. 


\title{
CIUDADANÍA Y EDUCACIÓN
}

Humberto Mejía Zarazúa*

\section{Resumen}

Esta aportación aborda la complejidad del concepto de ciudadanía mediante la mención de algunos de sus componentes y dimensiones; es decir, se considera que la noción de ciudadanía pierde simplicidad y se convierte en un actual campo de debate. Éste no debe quedar fuera del quehacer educativo en tanto la educación tiene, entre otras finalidades, la de formar ciudadanos críticos, reflexivos y transformadores de estructuras sociales, políticas y económicas que producen exclusión, desigualdad e inequidad entre los seres humanos. Así, es necesaria la transformación de las escuelas en instituciones democráticas para la formación de personas con amplias competencias ciudadanas.

\begin{abstract}
:
This contribution approaches the complexity of the concept of citizenship by means of the mention of some of its components and dimensions; that is to say, that the notion of citizenship is considered to lose simplicity and is becoming a present field of debate. It does not have to be left outside the educative task since education has, among other purposes, to form critical, reflective and transforming citizens of social, political and economic structures that produce exclusion, inequality and inequity between human beings. Thus, the transformation of schools in democratic institutions is necessary for the formation of people with wide citizen competence.
\end{abstract}




\section{La ciudadanía como concepto complejo}

La ciudadanía, como todo concepto tiene su historicidad. La concepción moderna la podemos ubicar en el siglo XIX en Europa, cuando el programa liberal buscó la identidad nacional al transformar los Estados en naciones y considerar a todos los habitantes que estaban dentro de las fronteras del Estado para convertirlos en ciudadanos. Sin embargo, se determinaron reglas para definirse como tal, "Las reglas variaban, pero siempre tendían a excluir (con más o menos rigor) a los recién llegados al Estado («migrantes»), a la vez que generalmente incluían a todos los considerados como residentes «normales»"(Wallerstein, 1998: 137). El lenguaje fue un importante elemento de diferenciación respecto de los Estados vecinos, y se impuso la adquisición de la lengua oficial a las minorías que manejaban otro lenguaje pero compartían el territorio de determinado Estadonación. Los modernos ciudadanos surgen de la tradición democrática francesa, inglesa y norteamericana, donde son ellos los que tienen el derecho a ejercer la soberanía del pueblo, aún cuando esto lleva implícito la división entre los ciudadanos y los no ciudadanos (extranjeros e inmigrantes por ejemplo).

Ángel Sermeño (2004) explica que la noción de ciudadanía tiene tres componentes: la posesión de un conjunto de derechos y deberes; la pertenencia a una comunidad política determinada (Estado-nación); y la capacidad de participar en la definición de la vida pública (política social y cultural). Jurídicamente la ciudadanía está definida por un conjunto de derechos y deberes obligatorios para 
los individuos que comparten un territorio denominado nación. En el plano políticoideológico la ciudadanía implica una identificación de comunidades que corresponden a la noción formal de nacionalidad. Para la sociología el concepto de ciudadanía implica relaciones de integración social, que a menudo producen fenómenos de exclusión en ámbitos comunitarios donde sus individuos aparentemente comparten condiciones de homogeneidad (Sojo, 2000). Los derechos individuales son inherentes al ciudadano según el concepto tradicional de ciudadanía expresado por Thomas $\mathrm{H}$. Marshall y conllevan una exigencia de derechos comunitarios. La ciudadanía es un estatus que implica una condición de igualdad entre los miembros de un Estado-nación (García Cabrera, 2002)

La concepción moderna de ciudadanía fue desarrollada fundamentalmente por el mencionado sociólogo británico T. H. Marshall, quien en 1950 publicó un trabajo titulado Citizenship and Social Class. Su concepto básicamente distingue tres dimensiones del concepto de ciudadanía: civil, política y social (Sojo, 2002). Esta división es importante desde el punto de vista metodológico ya que sienta las bases para la ubicación del concepto de ciudadanía en una escala de evolución histórica. Por supuesto que existen otros teóricos que han abordado la cuestión de la ciudadanía y que han actualizado y enriquecido las concepciones teóricas de Marshall de acuerdo con el proceso histórico mundial. Marshall estableció que "Ciudadanía es un status asignado a todos aquellos que son miembros plenos de una comunidad. Todos los que poseen dicho status son iguales con respecto a derechos y deberes" (Marshall citado por Sojo, 2002: 27). Las mencionadas dimensiones corresponden, según el autor, a un desarrollo secuencial, aspecto que le ha sido criticado ${ }^{[1]}$, aunque él mismo planteó que los traslapes son notorios. En general, los derechos civiles son la libertad de pensamiento, expresión, religión, derecho a la propiedad privada y a la justicia; los derechos 
políticos se refieren al derecho a la participación, como elector o con poder político; y los derechos sociales van desde el derecho al bienestar y seguridad económica hasta el derecho a la convivencia con la comunidad de pertenencia (Sojo, 2002). A este respecto Luis Carlos Ugalde, (2005: 30) al referirse al Informe del Programa de las Naciones Unidas para el Desarrollo (PNUD), denominado La Democracia en América Latina afirma que:

"La ciudadanía política comprende cuatro componentes: a) el derecho al voto; b) elecciones limpias; c) elecciones libres; d) elecciones como instrumento para acceder a cargos públicos.

La ciudadanía civil, por su parte, consta de cuatro piezas -según el informe- a) la igualdad legal y la protección contra la discriminación; b) el derecho a la vida, la integridad física y la seguridad; c) la administración de la justicia; d) la libertad de prensa y el derecho a la información.

Finalmente, la ciudadanía social se refiere -según el PNUD- a aquellos aspectos de la vida de los ciudadanos que afectan el potencial para desarrollar sus capacidades. Los derechos a la salud y a la educación son componentes básicos de la ciudadanía social. El desempleo, la pobreza y la desigualdad obstaculizan la integración de grandes grupos de individuos a la sociedad y constriñen la vigencia de la ciudadanía social."

Ángel Sermeño (2004: 89) señala que gracias a T. H. Marshall “...el componente de derechos ( $\mathrm{y}$ deberes) dominó ampliamente la reflexión sobre dicho concepto a lo largo de varias décadas (desde el inicio de la posguerra hasta el inevitable declive del Estado de Bienestar). En particular, en dicho contexto, hablar de ciudadanía era, con frecuencia y normalidad, referirse a las problemáticas vinculadas con la cuestión de las políticas de redistribución en el Estado de bienestar". Actualmente, la ciudadanía se relaciona con pertenencia y reconocimiento del sujeto, lo cual amplía y replantea el concepto mismo. 
Una idea más contemporánea de ciudadanía "emerge en el mundo de la posguerra fría [...] como manifestación de resistencia a un liberalismo presuntamente global y presuntamente universal, que no conoce como legítima la intervención del Estado en la regulación de las relaciones entre las personas y el mercado" (Sojo, 2002: 29), ésta es una concepción que tiene relación con la frontera entre la nociónformal de ciudadanía y la noción sustantiva. La noción formal se refiere a la pertenencia a un Estado-nación; y la sustantiva que se refiere a la capacidad de ejercicio de derechos públicos y privados, en las áreas que históricamente se han desarrollado alrededor del concepto de ciudadanía (Sojo, 2002). Sin embargo, si observamos la condición de las mujeres y los grupos étnicos subordinados, vemos que la ciudadanía formal no es condición suficiente ni necesaria para ejercer la ciudadanía sustantiva. Lo anterior se relaciona con el tipo de democracia de que se trate; en efecto, la ciudadanía se ejerce en la democracia, ésta, en una concepción moderna es igualación y equidad, implica un proceso autoinstituyente, aunque la democracia moderna tiene una deuda con la equidad, a pesar de la apertura política y social que representa la confluencia entre modernidad y democracia (Sojo, 2002).

La noción de ciudadanía es un campo de actual debate en tanto implica nociones de nosotros, elloso los otros. Es decir, se es ciudadano ante quienes no lo son. El concepto en sí encierra la contradicción de la pertenencia a un estatus y un valor universal, Ángel Sermeño (2004: 88) escribe que "la ciudadanía es, ciertamente, un reconocido estatus de inclusión y pertenencia que apela a la existencia de una estructura de derechos universales. Pero también es un proceso histórico, resultado de una diversidad de prácticas y/o dinámicas que a su vez han seguido su propio patrón, por llamarle de alguna manera, de interpretación nacional particular". 
Si bien el concepto de ciudadanía puede ser una mirada teórica del comportamiento humano, también es un ejercicio cotidiano, una práctica social (Sojo, 2002). Asimismo, el debate acerca de la ciudadanía cobra importancia en lo que respecta a la definición de las políticas públicas de cualquier país. Dicho concepto implica un ejercicio o práctica ciudadana que involucra aspectos políticos, económicos, sociales y culturales, que van desde el sufragio para la elección de gobernantes, hasta concepciones con respecto a antagonismos de género, étnicos y ambientales.

La revisión del concepto contemporáneo de ciudadanía en América Latina cobra importancia a partir de 1980, debido a la transición democrática más o menos generalizada en la región, toda vez que el tema está ligado a la forma democrática de gobierno y a la relación entre los individuos y el poder, así como entre el interés particular y el general, es decir, el tema de la justicia en términos de la tensión entre los derechos individuales y la pertenencia a una comunidad (Sojo, 2002; Sermeño, 2004). En suma, la noción de ciudadanía contiene complejidad conceptual y práctica.

El concepto de ciudadanía también adquiere complejidad en sus posibilidades de resignificación, puesto que hoy en día en que la especie humana está auto-amenazada es difícil tener claridad de lo que puede significar la ciudadanía y las nuevas solidaridades que son necesarias para la construcción de un mundo menos desigual para la humanidad. La comunidad de destino en el que todos estamos involucrados (Morin, 2002), implica la construcción de una identidad humana común, que podría significar la construcción de una ciudadanía planetaria, ya que es 
"la progresión y el anclaje de esta conciencia de pertenencia a nuestra patria terrestre son los que permitirán el desarrollo, por múltiples canales, en las diferentes regiones del globo, de un sentimiento de unión y de intersolidaridad necesaria para civilizar las relaciones humanas [...] Son el alma y el corazón de la segunda globalización, producto antagónico de la primera, los únicos que permitirán humanizar esta globalización" (Morin, 2002: 77)

Para lo cual la noción de ciudadanía y el ejercicio que conlleva en las esferas pública y privada deben concatenar la práctica solidaria y responsable en la dimensión local, nacional y planetaria.

\section{Educación y ciudadanía}

El conocimiento es fundamental para potenciar el desarrollo humano, tanto en el plano individual como en el social, por lo que es un instrumento para el ejercicio ciudadano en las distintas esferas de poder, entendiendo dicho ejercicio como la capacidad de los sujetos e instituciones para mantener o transformar su ambiente físico o social, así, el poder es estructural, condicionado y condicionante de las pautas de comportamiento social y cultural. La esfera de poder, concepto de David Held (1995: 212) "es un contexto de interacción o medio institucional en y a través del cual el poder da forma a las capacidades de las personas; es decir moldea y circunscribe sus perspectivas de vida y su participación efectiva en la elaboración de las decisiones públicas".

El concepto de esferas de poder nos permite ubicar terrenos de ejercicio ciudadano en una amplia perspectiva, es decir, trata de abarcar en lo posible los aspectos del "mundo de la vida" $\stackrel{[2]}{ }$. Así, un concepto amplio de poder permite 
ubicar un concepto amplio de ejercicio ciudadano, en virtud de que las relaciones de poder afectan el derecho a la autodeterminación de las personas, y por lo tanto de su autonomía y su condición ciudadana. Held (1995) explica que son varios planos donde puede vulnerarse la perspectiva de vida (seguridad y bienestar personal, participación política y social, identidad cultural, influencia sobre la agenda política y económica, etc.).

Francisco Miranda (2003) retoma a Held para proponer una educación que promueva el ejercicio ciudadano consciente, que influya en las diferentes esferas de poder:

"La educación debe ser, por lo tanto, un ámbito institucional que, en virtud del conocimiento que produce, asimila, transmite y distribuye, debe crear las oportunidades, alternativas de acceso, y resultados favorables, para hacer efectivo el ejercicio de los derechos ciudadanos en el ámbito de la salud, el bienestar social, la cultura, la participación cívica, la economía, las instituciones políticas y de las relaciones coercitivas y la violencia organizada" (Miranda, 2003: 52).

Uno de los fines de la educación es construir valores, éstos son:

"bienes que la persona puede hacer reales y concretos, hacer que pasen del nivel abstracto al nivel concreto de la actuación. Los valores son lo que mueve el actuar, sobre todo el moral, ya que lo que es valioso para la persona o la sociedad es lo que determina de alguna manera que se procure alcanzarlo con el proceso de la conducta." (Arriarán 1999: 42).

Los valores son el motor teleológico de las personas, en este sentido es importante promover "... los valores en que se sustentan la democracia social y política, que son el respeto al otro y a sus derechos, la tolerancia, la convivencia 
en una sociedad plural, la participación en los asuntos públicos, la responsabilidad por el bien común y la solidaridad" (Latapí 2003: 123). La formación de un ciudadano moderno, en el terreno pedagógico, implica una educación basada en el diálogo, lo cual es un reclamo de la modernidad, a fin de "desechar el monólogo y sus exclusivismos culturales, religiosos, económicos, sexuales, raciales, etc [...] Sólo si la praxis pedagógica se desenvuelve en medio de una situación de diálogo como apología de lo humano será posible un cambio efectivo del horizonte cultural, religioso y social del hombre" (Duch, 1997: 63). Se requiere de la superación del monólogo a partir de la descentración del yo, el reconocimiento de la alteridad y la diferencia.

En México ha sido importante la transición política (alternancia del partido en el poder si se quiere) para configurar un nuevo escenario en la formación valoral, en específico en la formación ciudadana y democrática. La educación en valores ciudadanos tiene un cariz político en tanto el importante papel que juega el Estado en dicha formación; y también son importantes las movilizaciones sociales fuera del espacio escolar en la dimensión de la vida cotidiana. Por otro lado, desde la innovación educativa Jaume Carbonell (2001: 14) asume que "La nueva ciudadanía que hay que formar exige desde los primeros años de la escolarización otro tipo de conocimiento y una participación más activa del alumnado en el proceso de aprendizaje".

La educación tiene la misión esencial de preparar a cada generación para ejercer la ciudadanía, ya que la razón fundante de crear el sistema escolar fue precisamente formar personas que respondieran a la conservación de determinado orden político y social. Ahora bien, actualmente se puede afirmar que 
la educación para la ciudadanía debe atravesar a todo el currículo. Aun cuando la escuela no sea, tal vez, el principal espacio para aprender la ciudadanía es indispensable para matizarla de una manera crítica y reflexiva, a través de lo que Kymlicka (2005: 54) Ilama el aprendizaje de la "razonabilidad pública", que en gran medida se desarrolla mediante "la presencia dentro del salón de clase de estudiantes con diferente origen étnico, religioso y cultural". Así, el proceso educativo, sea formal, informal o no formal, está indisolublemente ligado a la formación de ciudadanos: La práctica ciudadana, al igual que la educación, tienden a ser procesos permanentes a lo largo de la vida de las personas. Es prácticamente imposible disociar de las sociedades democráticas los conceptos de educación, ciudadanía y construcción del futuro. Al respecto Francisco Imbernón (2002: 5, 6) afirma:

"Sin educación no hay futuro; y si lo hay, es un futuro que condena a los pueblos, a los ciudadanos y las ciudadanas, a la alienación, a la explotación, a la dependencia y al sometimiento a otros. Hurtar a la humanidad el derecho a la educación es privarla de una de las herramientas más importantes que pueda tener a su disposición, la que le permita desarrollar la capacidad de emitir juicios y realizar acciones autónomas, de escoger y de razonar los motivos por los que ha hecho una elección u otra. Es hurtarle la capacidad de deliberar, debatir, comprender y aceptar las razones ajenas. Pero para ello la educación no debe limitarse a preparar personas cultas, sino que debe, sobre todo, educar para la ciudadanía".

La institución escolar históricamente ha vivido la tensión entre igualdad y libertad; aparato reproductor de ideología dominante y espacio liberador; instancia de carácter elitista y promotora del derecho universal a la educación. Aún así es una institución que contiene capital cultural, cierta autonomía moral y funcionalidad en la compensación de desigualdades sociales de origen. Las escuelas pedagógicas progresistas no son nuevas, tienen una larga tradición aunque 
frecuentemente han sido marginadas por el poder al enfatizar los procesos sociales cooperativos y democráticos, el compromiso con la transformación escolar y social, la vinculación con el entorno, una concepción globalizada del conocimiento, y la lucha por la igualdad social y el respeto a la individualidad. Por otro lado, es necesario desarrollar un pensamiento reflexivo y de comprensión histórica como medio para lograr una ciudadanía libre, culta y democrática. También es importante cultivar un conocimiento que mire al entorno para interpretarlo y transformarlo mediante proyectos de educación ambiental, consumo, salud, educación para la paz, interculturalismo, educación vial, medios de comunicación, educación afectivo-sexual, moral y cívica. Lo anterior se basa en la concepción del centro escolar como entidad donde el conocimiento se hace público y se democratiza la producción y distribución del saber, así como la reivindicación de la igualdad de oportunidades para todos los jóvenes mediante una escolarización de igual calidad (Carbonell, 2001).

La democracia es una forma de vida, J. Dewey (citado por Carbonell, 2001) sostuvo que sólo puede lograrse desde la educación y se basa en la razón, el método científico y la constante reorganización y reconstrucción de la experiencia, es decir, la democracia y la enseñanza son inseparables y se influyen mutuamente. En la medida en que la escuela y otras instituciones son más públicas y democráticas, la democracia social se fortalece. Una escuela democrática genera procesos de participación, diálogo y acuerdos; fomenta el derecho para intervenir en la toma de decisiones que afectan su vida institucional en articulación con el entorno social. "Apela a la formación de una ciudadanía activa, con ideas y proyectos propios, en contraste con la atonía, pasividad y mera supervivencia a que a menudo están sometidos los seres humanos a 
consecuencia de políticas autoritarias o democracias formales o maquilladas" (Carbonell, 2001: 93).

En una escuela democrática existe mayor atención a las necesidades e intereses del alumnado, se incrementa la interacción de los sujetos, se mejoran las condiciones de aprendizaje y la institución se compromete con la comunidad para tratar de combatir las desigualdades sociales de origen y sus causas. Desde la escuela se puede enseñar a interpretar de manera crítica las diversas informaciones y mensajes del entorno tanto inmediato como lejano, así como evitar localismos que estrechen la cosmovisión y encontrar la compatibilidad de lo

global con lo local, bajo la guía del respeto a los derechos humanos (Carbonell, 2001). Asimismo, tal como lo afirma Antonio Alanís (2000: 17) "Uno de los fines de la educación es que la sociedad en su conjunto cambie; no solamente que aprenda y/o aprehenda la cultura para que dicha cultura se rehaga y se transforme en nuevos códigos y hábitos culturales"

\section{Consideraciones finales}

El desarrollo educativo de los sujetos es necesario para la construcción de una nación independiente, justa y democrática; también representa un bien cultural fundamental para el incremento de la calidad de vida. La formación de ciudadanos comprometidos con la transformación social cobra una creciente importancia por varios motivos, uno de ellos es que al ir perdiendo la educación su carácter impulsor de movilidad social y la capacidad de equilibrar la concentración del ingreso y las estructuras sociales, la formación ciudadana es indispensable para asegurar, al menos, una participación más activa y consciente de los sujetos, individuales o colectivos, en la vida política, económica y social de la nación, que 
permita conformar horizontes promisorios en la construcción de un país más democrático, con menos desigualdad y exclusión. También cobran relevancia fundamental las prácticas educativas democráticas y solidarias, así como la participación para el bien común y el respeto a la diversidad. La educación es un proceso que potencia las posibilidades creativas del sujeto; además de informar, la educación debe formar, mirando al ser humano como un conjunto integrado único de potencialidades por desarrollar.

La transición hacia una educación más democrática, no se limita a formar ciudadanos que sólo hagan uso de sus derechos sociales, económicos y políticos otorgados por el Estado, sino que la práctica ciudadana representa una actividad capaz de transformar estructuras políticas, sociales y económicas. La educación democrática puede verse como un proceso propiciador de autonomía de individuos, grupos e instituciones; esta autonomía de sujetos, a su vez, se encuentra en función de las oportunidades de acceso tanto a bienes materiales como a bienes culturales, así como en el conocimiento y resignificación de los fenómenos, procesos y hechos que determinan a los sujetos, individuales y colectivos, y los convierten en aliados en la perpetuación del poder dominante

\section{Referencias}

ALANíS Huerta, Antonio (2000), Formación de formadores, México: trillas. 
ARRIARÁN, Samuel y Mauricio Beuchot (1999), Virtudes, Valores y Educación Moral. Contra el paradigma neoliberal, México: Universidad Pedagógica Nacional.

CARBONELL, Jaume (2001), La aventura de innovar. El cambio en la escuela, Madrid: Morata.

DUCH, Lluís (1997), La educación y la crisis de la modernidad, España: Paidos Educador.

GARCÍA Cabrero, Benilde (2002), Educación, ciudadanía y participación democrática, en: "Deconstruyendo la ciudadanía. Avances y retos en el desarrollo de la cultura democrática en México", México: SEGOB, SEP, IFE.

HABERMAS, Jürgen (2002), Teoría de la acción comunicativa, l. México: Taurus

HELD, David (1995). La democracia y el orden global. Del Estado moderno al gobierno cosmopolita, España: Paidós

KYMLICKA, Will (2005), La escuela y la formación del ciudadano, en "Educación 2001", núm. 125, octubre 2005. México.

LATAPÍ Sarré, Pablo (2003). El debate sobre los valores en la escuela mexicana, México: FCE.

MIRANDA, Francisco (2003), Necesidades humanas y educación. Una aproximación al concepto de desarrollo educativo. México: Praxis.

MORIN, Edgar (2002), La cabeza bien puesta. Repensar la reforma. Reformar el pensamiento, Buenos Aires: Nueva visión

O'DONELL, Guillermo (2004), "El debate conceptual sobre la democracia. Notas sobre la democracia en América Latina" en PNUD, La democracia en América Latina, Buenos Aires; Aguilar, Altea, Taurus, Alfaguara,

SERMEÑO, Ángel (2004), Ciudadanía y teoría democrática, en: "Metapolítica", Núm. 33Vol. 8, enero/febrero 2004.

SOJO, Carlos (2000), "La noción de ciudadanía en el debate latinoamericano", en Revista de la CEPAL 76". Pág. 25-37.

UGALDE, Luis Carlos (2005), El falso dilema entre desarrollo y democracia, en: nexos, año 27, Vol. XXVII, Num. 325, enero 2005, México.

WALLERSTEIN, Immanuel (1998), Después del liberalismo, México: siglo veintiuno editores, UNAM.

${ }^{11}$ Guillermo O' Donnell (2004: 55) aclara “...Gran Bretaña, Francia, Escandinavia y otros siguieron aproximadamente la secuencia postulada por T, H, Marshall. Esto es, primero el logro de la extensión significativa de derechos civiles, luego los políticos, y más tarde los sociales. En cambio, la secuencia Prusia/Alemania significó primero el logro de derechos civiles, luego derechos 
sociales y más tarde políticos [...] las secuencias del Noroeste se aplican de manera bastante aproximada a Costa Rica, Chile y Uruguay. Costa Rica y Chile siguieron el «patrón marshalliano» de derechos civiles-políticos-sociales [...] Esta no fue la ruta seguida por el resto de América Latina. Más bien, el patrón nodal ha sido el siguiente: primero se otorgaron algunos derechos sociales, más limitados que en el Noroeste, y en las últimas dos décadas en la mayoría de los países aquellos han sido profundamente revertidos. Más tarde, adquisición de derechos políticos, a través de procesos pasados o presentes de democratización política. Y tercero, aún hoy, derechos civiles implantados de manera sesgada e intermitente. Este es el patrón nacional-populista seguido por la Argentina, Bolivia, Brasil, Ecuador, México y Perú." (Resaltado del autor)

${ }^{[2]}$ En términos de Habermas (2002: 154) significa: “...aquellos objetos simbólicos que generamos cuando hablamos y actuamos, desde las manifestaciones inmediatas (como son los actos de habla, las actividades teleológicas, etc.) pasando por los sedimentos de tales manifestaciones (como son los textos, las tradiciones, los documentos, las obras de arte, las teorías, los objetos de la cultura material, los bienes, las técnicas, etc.) hasta los productos generados indirectamente, susceptibles de organización y capaces de estabilizarse a sí mismos (como son las instituciones, los sistemas sociales y las estructuras de personalidad)." 